\title{
COVID-19 and Guillain-Barre Syndrome Case Report
}

\author{
Amira Sidig ${ }^{1}$, Khabab Abbasher ${ }^{2}$, Hussien Abbasher ${ }^{3}$, Mohammed Abbasher ${ }^{4}$, and Abbasher Hussien ${ }^{5 *}$ \\ ${ }^{1}$ Associated professor of community medicine at Nelain University, faculty of medicine, Khartoum City, Sudan \\ ${ }^{2} \mathrm{MBBS}$ student at University of Khartoum Faculty of medicine, Khartoum City, Sudan \\ ${ }^{3}$ MBBS student at AlYarmouk College, Khartoum City, Sudan \\ ${ }^{4}$ MBBS student at Neilain University faculty of medicine, Khartoum, Sudan \\ ${ }^{5}$ Professor of Neurology at University of Khartoum, Faculty of medicine, Khartoum City, Sudan
}

*Corresponding author: Abbasher Hussien, Professor of Neurology at University of Khartoum, Faculty of medicine, Khartoum City, Sudan, E-mail: abbashar59@yahoo.com

Received: 19 May, 2020 | Accepted: 05 Jun, 2020 | Published: 30 Dec, 2020

Citation: Sidig A, Abbasher K, Abbasher H, Abbasher M, Hussien A (2020) COVID-19 and Guillain-Barre Syndrome Case Report. J Neurol Neurobiol $7(1)$ : dx.doi.org/10.16966/2379-7150.169

Copyright: (C) Sidig A, et al. This is an open-access article distributed under the terms of the Creative Commons Attribution License, which permits unrestricted use, distribution, and reproduction in any medium, provided the original author and source are credited.

\begin{abstract}
Introduction: Coronaviruses are a group of related viruses that cause diseases in mammals and birds. Guillain-Barre syndrome is a rare disorder in which the body's immune system attacks peripheral nerves.

The case: 65 years old Sudanese male not known to have diabetes mellitus or hypertension. When I have seen him he has upper and lower limbs weakness (quadriplegia). The condition was preceded by upper respiratory tract infection. Chest X-ray showed features of pneumonia Chest CT scan showed multiple bilateral ground glass opacities and consolidation typical of COVID-19 pneumonia. Brain MRI was normal. COVID-19 nasal swab test was positive. Nerve conduction study showed the evidence of polyradiculopathies with dominant demyelination supporting the diagnosis of Guillain-Barre syndrome. The patients died after seven days; because of progressive respiratory failure.
\end{abstract}

Keywords: COVID-19 and Guillain-Barre syndrome; COVID-19 case report; COVID-19 and Neurology; COVID-19 neurological manifestations

\section{Introduction}

Coronaviruses are a group of related viruses that cause diseases in mammals and birds. Human to human transmission of coronaviruses is primarily thought to occur among close contacts via respiratory droplets generated by sneezing and coughing [1].

Pneumonia of unknown cause detected in Wuhan, China was first reported to the WHO Country Office in China on 31 December 2019. Most people infected with the COVID-19 virus will experience mild to moderate respiratory illness and recover without requiring special treatment. Older people and those with underlying medical problems like cardiovascular disease, diabetes, chronic respiratory disease, and cancer are more likely to develop serious illness. People may be sick with the virus for 1 to 14 days before developing symptoms [2].

The most common symptoms of coronavirus disease (COVID-19) are cough, shortness of breath or difficulty breathing, fever, chills, muscle pain, sore throat and new loss of taste or smell. Most people (about $80 \%$ ) recover from the disease without needing special treatment. The disease can be serious and even fatal $[3,4]$

Covid-19 can cause serious respiratory problems such as respiratory failure, acute respiratory distress syndrome [5]. As was reported by Brit long and his colleagues Coronavirus can cause a number of cardiovascular complications like myocardial injury and myocarditis, acute myocardial infarction, acute heart failure and cardiomyopathy, dysrhythmias and venous thromboembolic events [6].

Some people suffering with severe cases of COVID-19 are showing signs of kidney damage [7]. Coronaviruses are not always confined to the respiratory tract, and under certain conditions they can invade the central nervous system and cause neurological pathologies. Neurological symptoms have been reported in patients affected by COVID-19, such as headache, dizziness, myalgia and anosmia, as well as cases of encephalopathy, encephalitis, necrotising haemorrhagic encephalopathy, stroke, epileptic seizures $[8,9]$.

\section{Guillain-Barre Syndrome}

It is a rare disorder in which the body's immune system attacks peripheral nerves. Weakness and tingling of the extremities are usually the first symptoms. These sensations can quickly spread, eventually paralyzing the whole body. The exact cause of GuillainBarre syndrome isn't known. The disorder usually appears days or weeks after a respiratory or digestive tract infection. Guillain-Barre syndrome may be triggered by: 
- Most commonly, infection with campylobacter

- Influenza virus

- Cytomegalovirus

- $\quad$ Epstein-Barr virus

- Zika virus

- Hepatitis A, B, C and E

- HIV, the virus that causes AIDS

- Mycoplasma pneumonia

- $\quad$ Surgery

- Trauma

- Hodgkin's lymphoma

- And rarely, influenza vaccinations or childhood vaccinations.

The weakness in Guillain-Barre syndrome usually starts in the distal parts of both lower limbs and spread to involve the proximal parts, trunk and both upper limbs. Cranial nerves may be involved; mainly bilateral facial pulses. Sensation usually is intact, although some patients may be stocks and gloves sensory disturbance. 3-5\% may have urine retention. There are two serious complications associated with Guillain-Barre syndrome:

1. Respiratory muscles paralysis

2. Autonomic nervous system involvement.

On clinical examination of patients with Guillain-Barre syndrome in addition to the weakness, hypotonia and areflexia are the dominant findings.

\section{Diagnosis}

Guillain-Barre syndrome can be difficult to diagnose in its earliest stages. Its signs and symptoms are similar to those of other neurological disorders and may vary from person to person. Investigations like lumbar puncture, electromyography and nerve conduction studies can help in diagnosis.

\section{Treatment}

There's no cure for Guillain-Barre syndrome. But two types of treatments can speed recovery and reduce the severity of the illness:

1. Plasma exchange (plasmapheresis).

2. L-iv immunoglobulin [10].

\section{Case Report}

\section{History}

65 years old Sudanese male not known to have diabetes mellitus or hypertension. When I have seen him he had upper and lower limbs weakness (quadriplegia). The condition was preceded by upper respiratory tract infection in form of low grade fever, sore throat, dry irritant cough, headache and generalized fatigability. After five days he started to complain of numbness and weakness involving both upper and lower limbs. It started distally and after three days the proximal parts of both lower limbs was involved. Three days later both upper limbs were affected. Also I noticed that he has facial paraesthesia with inability to close his mouth and his both eyes. Also he reported that he has clumsiness of both upper limbs. This was followed by urinary incontinence. Then he became completely paralyzed. He has no chest pain, palpitations or dyspnea. The condition was not associated with convulsions or loss of consciousness. During the course of the disease he developed breathlessness and we thought of respiratory muscles involvement and autonomic nervous system involvement. When he was investigated by (CT chest) it raised the suspension of COVID-19 infection and when he was investigated for COVID-19 the results was positive. Then he was transferred to the ICU where he received both IV immunoglobulin and connected to mechanical ventilator.

\section{Examination}

On examination the patient looked ill and pale, but not jaundiced or cyanosed. He has no chest pain, palpitations or dyspnea. His pulse was $78 /$ minute, regular with good volume. His blood pressure was 110/70 $\mathrm{mmH}$. Examination of the cardiovascular system, chest and abdomen was normal. The abnormalities were confined to the central nervous system. The patient was fully conscious and oriented in time, person and place. Both his recent and remote memory was intact. Although he was a little pit alert, but he was co-operative.

Cranial nerves examination revealed an evidence of olfactory nerves involvement and bilateral papilledema. There was bilateral facial nerves involvement and slight palatal muscle weakness. There was no neck weakness. He has truncal weakness. Both upper and lower limbs examinations showed no wasting or deformity. There was generalized hypotonia and areflexia. Power was grade three in all groups of muscles. All modalities of sensation were intact including light touch, pinprick and vibration. Coordination cannot be demonstrated due to the weakness. Lists of investigations were done including urine analysis, stool analysis, blood urea, electrolyte and liver function test and all were normal. ECG and echocardiography were normal. Chest X-ray showed features of pneumonia. Chest CT scan showed multiple bilateral ground glass opacities and consolidation typical of COVID-19 pneumonia. Brain MRI was normal. COVID-19 nasal swab test was positive. Nerve conduction study showed the evidence of polyradiculopathies with dominant demyelination supporting the diagnosis of Guillain-Barre syndrome.

The patients died after seven days; because of progressive respiratory failure.

\section{Discussion}

The full detailed history and proper clinical examination in addition to the nerve conduction study findings are in favour of acute polyradiculoneuritis with prominent demyelinating features, so of a diagnosis acute Guillain-Barre syndrome associated with COVID-19 was made. Guillain-Barre syndrome is an acute/subacute immune mediated polyradiculoneuropathy characterized by vary degrees of limbs weakness and sometimes cranial nerves involvement. There is hypotonia and areflexia in the affected limbs in addition to the weakness. Also it can present with sensory disturbances in the form of stocks and gloves impairment of sensation. In few numbers of patients there may be urinary incontinence, respiratory muscles paralysis and autonomic nervous system involvement.

Corona virus infection may be asymptomatic. The most common symptoms at the onset of the disease are generalized fatigability, headache, low grade fever and sore throat [11-13]. Also it can present with GIT symptoms (diarrhea) [14]. Systemic complications of COVID-19 infection are acute cardiac damage, renal failure, recurrent pulmonary embolism and acute respiratory distress syndrome $[15,16]$. Almost 35\% of patients with COVID-19 infection present with neurological manifestations like headache, loss of taste and smell, myopathy, epilepsy, stroke (hemorrhagic or infarction) and encephalitis $[17,18]$. Corona virus may cause neurological disorders through its direct effect on the brain or by activation of the immune system [19]. 
The mechanism by which COVID-19 causes Guillain-Barre syndrome was not yet investigated but it was known that Coronavirus stimulates inflammatory cells and produce inflammatory cytokinase, this creates immunomediated process, but it was not known whether Covid-19 induces the production of antibodies against specific gangliosides like what tends to occur with curtain forms of Guillain-Barre syndromes [20-21].

Limitations of this report are lack of screening of autoimmune disease, paraneoplastic syndrome, vasculitis relating antibodies, and antigangliosides testing.

\section{Recommendations}

- We recommend that during the course of treatment of COVID-19 infection a close monitoring of the neurological complications of the disease is needed.

- The relationship between Covid-19 infection and GuillainBarre syndrome needs to be confirmed in large case series; so early recognition and treatment of the neuromuscular and autonomic worsening leading to cardio respiratory failure in patients with Guillain-Barre syndrome and mild pulmonary COVID-19 can be achieved.

\section{References}

1. Shereen MA, Khan S, Kazmi A, Bashir N, Siddique R (2020) COVID-19 infection: Origin, transmission, and characteristics of human coronaviruses. J Adv Rese 24: 91-98.

2. World Health Organization (2020) Rolling updates on coronavirus disease (COVID-19)

3. Coronavirus Disease (COVID-19) (2019) People Who Are at Higher Risk for Severe Illness. Centers for Disease Control and Prevention.

4. COVID-19 and Gastrointestinal Symptoms.

5. Complications Coronavirus Can Cause.

6. Long B, Brady WJ, Koyfman A, Gottlieb M (2020) Cardiovascular Complications in COVID-19. Am J Emerg Med S0735-6757(20)30277-1.

7. Coronavirus: Kidney Damage Caused by COVID-19. Johns Hopkins medicine.

8. Carod-Artal FJ (2020) Neurological Complications of Coronavirus and COVID-19. Rev Neurol 70: 311-322.
9. Mao L, Jin H, Wang M, Hu Y, Chen S, et al. (2020) Neurologic Manifestations of Hospitalized Patients with Coronavirus Disease 2019 in Wuhan, China. JAMA Neurol e201127.

10. Guillain-Barre syndrome. Mayo Clinic.

11. Casadevall A, Pirofski LA (2020) The Convalescent Sera Option for Containing COVID-19. J Clin Invest 130: 1545-1548.

12. Wang D, Hu B, Hu C, Zhu F, Liu X, et al. (2020) Clinical Characteristics of 138 Hospitalized Patients with 2019 Novel Coronavirus-Infected Pneumonia in Wuhan, China. JAMA 323: 1061-1069.

13. Li YC, Bai WZ, Hashikawa T (2020) The neuroinvasive potential of SARS-CoV2 may play a role in the respiratory failure of COVID-19 patients. J Med Virol 92: 552-555.

14. Krauer F, Riesen M, Reveiz L, Oladapo TO, Martínez-Vega R, et al. (2017) Zika Virus Infection as a Cause of Congenital Brain Abnormalities and Guillain-Barré Syndrome: Systematic Review. PLoS Med 14: e1002203.

15. McGonagle D, Sharif K, O'Regan A, Bridgewood C (2020) The Role of Cytokines including Interleukin-6 in COVID-19 induced Pneumonia and Macrophage Activation Syndrome-Like Disease. Autoimmun Rev 2020: 102537.

16. Qin C, Zhou L, Hu Z, Zhang S, Yang S, et al. (2020) Dysregulation of immune response in patients with COVID-19 in Wuhan, China. Clin Infect Dis Epub ciaa248.

17. Mao L, Wang M, Chen S, He Q, Chang J, et al. (2020) Neurological Manifestations of Hospitalized Patients with COVID-19 in Wuhan, China: a retrospective case series study. medRxiv.

18. Sejvar JJ, Baughman AL, Wise M, Morgan OW (2011) Population Incidence of Guillain-Barré Syndrome: A Systematic Review and Meta-Analysis. Neuroepidemiology 36: 123-133.

19. Jacobs $B C$, Rothbarth PH, van der Meché FG, Herbrink $P$, Schmitz $\mathrm{PI}$, et al. (1998) The Spectrum of Antecedent Infections in GuillainBarré Syndrome: A Case-Control Study. Neurology 51: 1110-1115.

20. Sahin AR, Erdogan A, Agaoglu PM, Dineri Y, Cakirci AY, et al. (2020) 2019 Novel Coronavirus (COVID-19) Outbreak: A Review of the Current Literature. EJMO 4: 1-7.

21. Kim JE, Heo JH, Kim HO, Song SH, Park SS, et al. (2017) Neurological Complications during Treatment of Middle East Respiratory Syndrome. J Clin Neurol 13: 227-233. 